\title{
Gender differences in the association between pre-adolescent smoking initiation and emotional or behavioural problems
}

\author{
Gea de Meer ${ }^{1,2^{*}}$, Matty R Crone ${ }^{3}$, Sijmen A Reijneveld ${ }^{2,3}$
}

\begin{abstract}
Background: Emotional and behavioural problems are a risk factor for the initiation of smoking. In this study, we aimed to assess this relationship beyond clinical cut-off values of problem behaviour.

Methods: Cross-sectional national survey among 9-13 year old children with data on smoking and Childhood Behaviour Checklist (CBCL) ( $\mathrm{N}=960)$. Relationships between smoking and tertiles of CBCL-scores were assessed.

Results: Smoking was reported by $5.9 \%$ of the children (7.1\% boys and $5.0 \%$ girls, $\mathrm{P}>0.100)$. Relationships between smoking and problem behaviour were present in girls, but ot in boys. Among girls, smoking was associated with attention problems, thought problems, and delinquent behaviour. For attention problems and delinquent behaviour the associations were limited to the CBCL-scores in the uppermost $16 \%$ which agrees with the subclinical cut-off value.

Conclusion: Pre-adolescent girls more frequently smoke if having attention problems, delinquent behaviour, or thought problems.
\end{abstract}

\section{Background}

Smoking-related diseases are the most common cause of death in Western societies and affluent countries [1]. Almost all adult smokers started smoking during adolescence [2]. Starting smoking at young age has been shown a risk factor for daily smoking and dependence [3].

There is a great amount of evidence for the cooccurrence of smoking initiation and emotional or behavioural problems [4-9]. Therefore, smoking prevention might benefit from targeting children with problem behaviour. So far, however, it is unclear if smoking prevention would benefit most from improvement of psychosocial health of all children, or from a targeted approach on children with more severe behavioural problems. Previous studies mostly relied on a dichotomous definition of problem behaviour like a clinical diagnosis [4-7], or the clinical or subclinical score on the Childhood Behaviour Checklist (CBCL), as reflected by respectively the uppermost $10 \%$ and

\footnotetext{
* Correspondence: g.demeer@hvdfryslan.nl

${ }^{1}$ Municipal Health Service Fryslân, Leeuwarden, the Netherlands

Full list of author information is available at the end of the article
}

$15 \%$ scores in the population $[8,9]$. This, however, does not give information on CBCL-scores below the subclinical cut-off value.

In this community-based study, we studied the relationship between smoking initiation and problem behaviour beyond subclinical cut-off values among pre-adolescent elementary school children. In addition, we searched for gender differences in the relationship between behaviour problems and the uptake smoking. Previous studies have shown that girls more frequently take up smoking in early adolescence [10,11]. Gender differences in the association with problem behaviour may play a role, as suggested by Rohde et al [12]. Therefore, we studied relationships between pre-adolescent problem behaviour and the uptake of smoking for boys and girls separately.

\section{Methods}

\section{Study protocol}

The study was embedded in Preventive Child Healthcare $(\mathrm{PCH})$ that provides health monitoring for all 0-19 yearold children according to Dutch public health legislation. Parents and their children are invited for regular examination that includes monitoring of development 
and growth, and screening activities (for example congenital disease, vision impairment, hearing loss). Participation rate to $\mathrm{PCH}$ routine examinations exceeds $90 \%$ without differences by gender, parental education, family income, and ethnic background [13].

Postal questionnaires were sent to a national sample of parent-child couples together with the invitation for a routine health examination by $\mathrm{PCH}$. Parents completed questions on background characteristics, the child's physical health, and behaviour problems. The child questionnaire comprised data on smoking, bullying, and friends. Parents and children completed the questionnaires at home and delivered them in a sealed envelope at the $\mathrm{PCH}$ clinic when coming for the child's preventive health examination. The study protocol was approved by the local Medical Ethical Committee including verbal informed consent by parents to the child health nurse that took in the sealed questionnaires.

\section{Study population}

The study population was obtained by means of a twostep procedure. First, a random sample of child-healthcare services was drawn, after stratification by region, urbanization ethnicity. Subsequently, for each PCH clinic a random sample of 100 children, grade 5-8 of primary school (age 7-13). After correction for stratified sampling, the samples were representative for the entire Dutch population in terms of income, type of family and parental educational level.

Out of the 1706 invited children 1350 (79\%) parents gave informed consent for participation in the study. Most important reason for non-participation was a lack of interest in the study (37\%). Children with missing data on $\operatorname{sex}(\mathrm{N}=46)$ or age $(\mathrm{N}=4)$ were excluded. Since smoking did not occur among children under 9 years of age, we restricted the population to children aged 9 and older $(\mathrm{N}=1230)$.

\section{Background characteristics}

Parents completed questionnaire data on the child's and family's background characteristics including sex, family composition, ethnic origin, parental education, and family income.

\section{Smoking}

Children were asked if they ever had smoked cigarettes, categorized as 'never', 'once or twice', 'regular', or 'daily'. For the current analysis we dichotomized the answers in 'never' and 'ever', since only 4 children reported 'regular' or 'daily' smoking.

\section{Child problem behaviour}

Parents completed the 120 problem items of the validated Dutch version of the Child Behaviour CheckList
(CBCL) $[14,15]$. For the current analysis, the CBCL smoking item was deleted. Raw CBCL scores were computed for eight syndrome scales, i.e. attention problems, aggressive behaviour, anxious/depressed, delinquent behaviour, social problems, somatic complaints, thought problems, and being withdrawn. Broadband groups of syndrome scales comprised internalizing (anxious/ depressed, somatic complaints, withdrawn) and externalizing problems (aggressive or delinquent behaviour). All CBCL-scores were categorized in tertiles of the genderspecific distribution indicating three levels of problem behaviour.

\section{Statistical analysis}

Statistical analyses were performed using the SPSS 12.0 statistical software package. Relationships between smoking initiation and tertiles of CBCL-scores were tested using Chi-square tests. Crude and multiple logistic regression analyses were performed. To explore differences by gender we performed stratified logistic regression analyses for sex.

\section{Results}

Of the 1230 children aged 9-13 years, the CBCL was completed for 1118 children and additional data on smoking were available for 960 children (78.0\%). Ever smoking was reported by 57 (5.9\%) children. Children that reported ever smoking were older $(\mathrm{P}=0.022)$ and less frequently had a highly-educated mother $(\mathrm{P}=0.026)$ compared to never smokers. There was no difference between ever and never smokers for sex, ethnic background, family composition, family size, and father's education (table 1 ).

Table 2 shows the distribution of smokers by tertiles of CBCL score. Among girls, a positive trend was observed for a report of smoking and a CBCL score for total problems $(P=0.062)$, delinquent behaviour $(P=0.050)$, attention problems $(\mathrm{P}=0.010)$, and thought problems $(\mathrm{P}=0.039)$. Except for thought problems, the higher prevalence rates only occurred for the uppermost tertiles of CBCL-scores. For boys we did not observe any relationships of smoking with CBCL-scores.

Logistic regression analyses adjusted for age, sex and mother's education yielded statistically significant odds ratios for thought problems and delinquent behaviour (table 3). Gender stratified logistic regression were performed for associations with $\mathrm{P}<0.100$ in bivariate (table 2 ) or multivariate analyses (table 3 ), i.e. total problems, delinquent behaviour, attention problems and thought problems. In boys, none of the CBCL problem scores was associated with smoking initiation (data not shown).

In girls, taking up smoking occurred more frequently in girls with a higher CBCL problem score for thought problems, attention problems, or delinquent behaviour (table 4). 
Table 1 Socio-demographic characteristics of smoking and non-smoking children

\begin{tabular}{|c|c|c|c|c|}
\hline & \multirow{2}{*}{$\begin{array}{c}\text { All } \\
(\mathrm{N}=960)\end{array}$} & \multicolumn{2}{|c|}{ Ever smoking } & \multirow[b]{2}{*}{ P-value } \\
\hline & & $\begin{array}{c}\text { No } \\
(\mathrm{N}=903)\end{array}$ & $\begin{array}{c}\text { Yes } \\
(\mathrm{N}=57) \\
\end{array}$ & \\
\hline \multicolumn{5}{|l|}{ Gender, N = 960: } \\
\hline Boy & $468(49 \%)$ & $435(48 \%)$ & $33(58 \%)$ & \\
\hline Girl & $492(51 \%)$ & 468 (52\%) & $24(42 \%)$ & 0.173 \\
\hline \multicolumn{5}{|l|}{ Age, $N=960$ : } \\
\hline $9-10$ years & $341(36 \%)$ & $329(36 \%)$ & $12(21 \%)$ & \\
\hline $11-13$ years & $619(64 \%)$ & $574(64 \%)$ & 45 (79\%) & 0.022 \\
\hline \multicolumn{5}{|l|}{ Ethnic origin, $N=960$} \\
\hline Dutch & $700(73 \%)$ & $653(72 \%)$ & $47(82 \%)$ & \\
\hline Non-Dutch & $260(27 \%)$ & $250(28 \%)$ & $10(18 \%)$ & 0.123 \\
\hline \multicolumn{5}{|l|}{ Family composition, N = 946} \\
\hline 2 parents & $849(90 \%)$ & $801(90 \%)$ & $48(87 \%)$ & \\
\hline 1 parent & $97(10 \%)$ & $90(10 \%)$ & $7(13 \%)$ & 0.494 \\
\hline \multicolumn{5}{|l|}{ Siblings, $N=960$} \\
\hline 0 or 1 & $554(58 \%)$ & $518(57 \%)$ & $36(63 \%)$ & \\
\hline 2 or more & $406(42 \%)$ & $385(43 \%)$ & $21(37 \%)$ & 0.411 \\
\hline \multicolumn{5}{|l|}{ Mother's education, $N=931$} \\
\hline University or high vocational & $198(21 \%)$ & $182(21 \%)$ & $16(29 \%)$ & \\
\hline Intermediate vocational & $272(29 \%)$ & $251(29 \%)$ & $21(37 \%)$ & \\
\hline Low vocational or high school & $461(50 \%)$ & $442(50 \%)$ & $19(34 \%)$ & 0.026 \\
\hline \multicolumn{5}{|l|}{ Father's education, $N=891$} \\
\hline University or high vocational & $254(26 \%)$ & $239(28 \%)$ & $15(29 \%)$ & \\
\hline Intermediate vocational & $242(27 \%)$ & $222(27 \%)$ & $20(28 \%)$ & \\
\hline Low vocational or high school & $395(44 \%)$ & $378(45 \%)$ & $17(33 \%)$ & 0.289 \\
\hline
\end{tabular}

Aiming at a more precise assessment of the relationships within the uppermost tertile of CBCL scores for attention problems and delinquent behaviour, logistic regression was repeated for split groups of the uppermost tertile $\left(67^{\text {th }}-83^{\text {rd }}\right.$ and $>83^{\text {rd }}$ percentiles $)$. To increase statistical power, the lowest and intermediate tertiles were combined as the reference group. As shown in Figure 1, for attention problems and delinquent behaviour only the uppermost $16.7 \%$ values of CBCL-scores were associated with ever smoking $(\mathrm{P}<0.050)$.

\section{Discussion}

In this study, we found that among pre-adolescent girls smoking initiation was associated with delinquency, attention problems, and thought problems. For delinquency and attention problems, this was limited to CBCL-scores in the subclinical and clinical range. In contrast, the association of smoking initiation with thought problems applied to mild problems as well. In boys smoking initiation was not associated with problem behaviour.

Our results confirms a relationship of smoking with attention deficit $[16,17]$, conduct problems $[4,7,18,19]$, delinquency and thought problems [8]. To our knowledge this is the first study that took into account the distribution of the data on child behaviour by analyzing in tertiles. Theoretically, this provides information on the role of problem behaviour beyond clinical cut-off values. Interestingly, our final results show that an association with smoking conferred the uppermost $16.7 \%$ of CBCL scores, i.e. close to the subclinical cut-off value of the uppermost $15 \%$ CBCL scores $[14,15]$.

Our results thus suggest that smoking prevention will benefit most from targeting a 'high-risk' group of children with behavioural problems. The association of smoking initiation with specific domains of problem behaviour may be taken into consideration as an additional reason for a 'high-risk' group approach. This may be added to general population strategies for smoking prevention, thereby increasing its effect in the subgroup of children with behavioural problems. To our opinion, this is a relevant group for smoking prevention since early starters have been shown at high risk of regular use, dependence and abuse later in life $[6,20]$.

In this study, we did not collect data on possible explanatory factors for the relationship between behavioural problems and cigarette smoking. Peer group pressure may play a role in children with delinquent 
Table 2 Number of ever smokers by tertiles of the distribution for CBCL

\begin{tabular}{|c|c|c|c|c|c|c|c|}
\hline & \multicolumn{6}{|c|}{ Tertile of CBCL score } & \multirow[b]{3}{*}{$\mathrm{p}$-value trenc } \\
\hline & \multicolumn{2}{|r|}{ Low } & \multicolumn{2}{|r|}{ Intermediate } & \multicolumn{2}{|r|}{ High } & \\
\hline & $\mathrm{N}$ & Ever smokers & $\mathrm{N}$ & Ever smokers & $\mathrm{N}$ & Ever smokers & \\
\hline \multicolumn{8}{|l|}{ Girls } \\
\hline Total problems & 170 & $6(4 \%)$ & 159 & $5(3 \%)$ & 163 & $13(8 \%)$ & 0.062 \\
\hline Externalizing problems & 146 & $6(4 \%)$ & 190 & $7(4 \%)$ & 156 & $11(7 \%)$ & 0.211 \\
\hline Internalizing problems & 194 & $8(4 \%)$ & 154 & $10(6 \%)$ & 144 & $6(4 \%)$ & 0.911 \\
\hline Aggressive behaviour & 168 & $8(5 \%)$ & 179 & $6(3 \%)$ & 145 & $10(7 \%)$ & 0.412 \\
\hline Delinquent behaviour & 245 & $8(3 \%)$ & 106 & $5(5 \%)$ & 141 & $11(8 \%)$ & 0.050 \\
\hline Anxious/depressed & 133 & $4(3 \%)$ & 211 & $11(5 \%)$ & 148 & $9(6 \%)$ & 0.237 \\
\hline Somatic complaints & 150 & $7(5 \%)$ & 178 & $7(4 \%)$ & 164 & $10(6 \%)$ & 0.544 \\
\hline Withdrawn & 205 & $8(4 \%)$ & 120 & $6(5 \%)$ & 167 & $10(6 \%)$ & 0.352 \\
\hline Attention problems & 189 & $4(2 \%)$ & 186 & $10(5 \%)$ & 117 & $10(8 \%)$ & 0.010 \\
\hline Social problems & 162 & $10(6 \%)$ & 128 & $5(4 \%)$ & 125 & $7(6 \%)$ & 0.783 \\
\hline Thought problems & 208 & $4(2 \%)$ & 126 & $10(8 \%)$ & 158 & $10(6 \%)$ & 0.039 \\
\hline \multicolumn{8}{|l|}{ Boys } \\
\hline Total problems & 163 & $9(6 \%)$ & 156 & $15(10 \%)$ & 149 & $9(6 \%)$ & 0.827 \\
\hline Externalizing problems & 158 & $13(8 \%)$ & 167 & $11(7 \%)$ & 143 & $9(6 \%)$ & 0.508 \\
\hline Internalizing problems & 155 & $11(7 \%)$ & 164 & $11(7 \%)$ & 149 & $11(7 \%)$ & 0.925 \\
\hline Aggressive behaviour & 137 & $10(7 \%)$ & 182 & $13(7 \%)$ & 149 & $10(7 \%)$ & 0.845 \\
\hline Delinquent behaviour & 158 & $9(6 \%)$ & 198 & $15(8 \%)$ & 112 & $9(8 \%)$ & 0.437 \\
\hline Anxious/depressed & 129 & $10(8 \%)$ & 177 & $14(8 \%)$ & 162 & $9(6 \%)$ & 0.445 \\
\hline Somatic complaints & 164 & $9(6 \%)$ & 185 & $13(7 \%)$ & 119 & $11(9 \%)$ & 0.227 \\
\hline Withdrawn & 171 & $12(7 \%)$ & 182 & $13(7 \%)$ & 115 & $8(7 \%)$ & 0.990 \\
\hline Attention problems & 141 & $10(7 \%)$ & 170 & $9(5 \%)$ & 157 & $14(9 \%)$ & 0.516 \\
\hline Social problems & 150 & $11(7 \%)$ & 177 & $10(6 \%)$ & 141 & $12(8 \%)$ & 0.708 \\
\hline Thought problems & 189 & $11(6 \%)$ & 113 & $11(10 \%)$ & 166 & $11(7 \%)$ & 0.737 \\
\hline
\end{tabular}

behaviour [21]. In children with attention problems, self-medication may contribute to the development of nicotine dependence since tobacco is known to ameliorate symptom severity, and enhance general attention [22-24]. Self-medication may also apply to thought problems as shown in schizophrenic patients $[25,26]$. We,

Table 3 Odds ratios and $95 \%$ confidence intervals for tertiles of CBCL-scores; adjusted for age, mother's education, and sex

\begin{tabular}{lccc}
\hline & \multicolumn{3}{c}{ Tertile of CBCL-score } \\
\cline { 2 - 4 } & Low (reference) & Intermediate & High \\
\hline Total problems & 1.0 & $1.4(0.7 ; 2.9)$ & $1.9(09 ; 3.7)$ \\
Externalising problems & 1.0 & $0.8(0.4 ; 1.7)$ & $1.3(0.7 ; 2.5)$ \\
Internalising problems & 1.0 & $1.2(0.6 ; 2.4)$ & $1.2(0.6 ; 2.4)$ \\
Aggressive behaviour & 1.0 & $0.9(0.5 ; 1.9)$ & $1.3(0.7 ; 2.5)$ \\
Delinquent behaviour & 1.0 & $1.7(0.8 ; 3.3)$ & $2.2(1.1 ; 4.4)$ \\
Anxious/depressed & 1.0 & $1.1(0.6 ; 2.1)$ & $1.2(0.6 ; 2.5)$ \\
Somatic complaints & 1.0 & $1.2(0.6 ; 2.2)$ & $1.4(0.7 ; 2.8)$ \\
Withdrawn & 1.0 & $1.1(0.6 ; 2.1)$ & $1.7(0.8 ; 3.3)$ \\
Attention problems & 1.0 & $1.6(0.8 ; 3.1)$ & $1.8(0.9 ; 3.5)$ \\
Social problems & 1.0 & $1.0(0.4 ; 2.3)$ & $1.4(0.8 ; 2.6)$ \\
Thought problems & 1.0 & $2.8(1.4 ; 5.5)$ & $1.7(0.8 ; 3.3)$ \\
\hline
\end{tabular}

however, emphasize that 'thought problems' in this study is not synonymous with psychiatric pathology. CBCL items for thought problems address problems with reality testing and obsessive/compulsive behaviour, though without a relationship with DSM-diagnoses. Since we did not collect data on any action to relieve symptoms, explanations with respect to self-medication for our results should be considered speculative. Nevertheless, our results are in agreement with previous studies that showed an earlier initiation of smoking in subjects with attention deficit or thought problems including schizophrenia $[12,27,28]$. It might be that children with symptoms of attention deficit or thought

Table 4 Odds ratios and $95 \%$ confidence intervals for tertiles of CBCL-scores in girls; adjusted for age and mother's education

\begin{tabular}{lccc}
\hline & \multicolumn{3}{c}{ Tertile of CBCL-score } \\
\cline { 2 - 4 } & Low (reference) & Intermediate & High \\
\hline Total problems & 1.0 & $0.9(0.3 ; 3.1)$ & $2.7(1.0 ; 7.5)$ \\
Delinquent behaviour & 1.0 & $1.6(0.5 ; 4.9)$ & $2.8(1.1 ; 7.3)$ \\
Attention problems & 1.0 & $3.1(0.9 ; 10.3)$ & $3.6(1.1 ; 11.8)$ \\
Thought problems & 1.0 & $4.5(1.4 ; 14.9)$ & $3.7(1.1 ; 12.1)$ \\
\hline
\end{tabular}




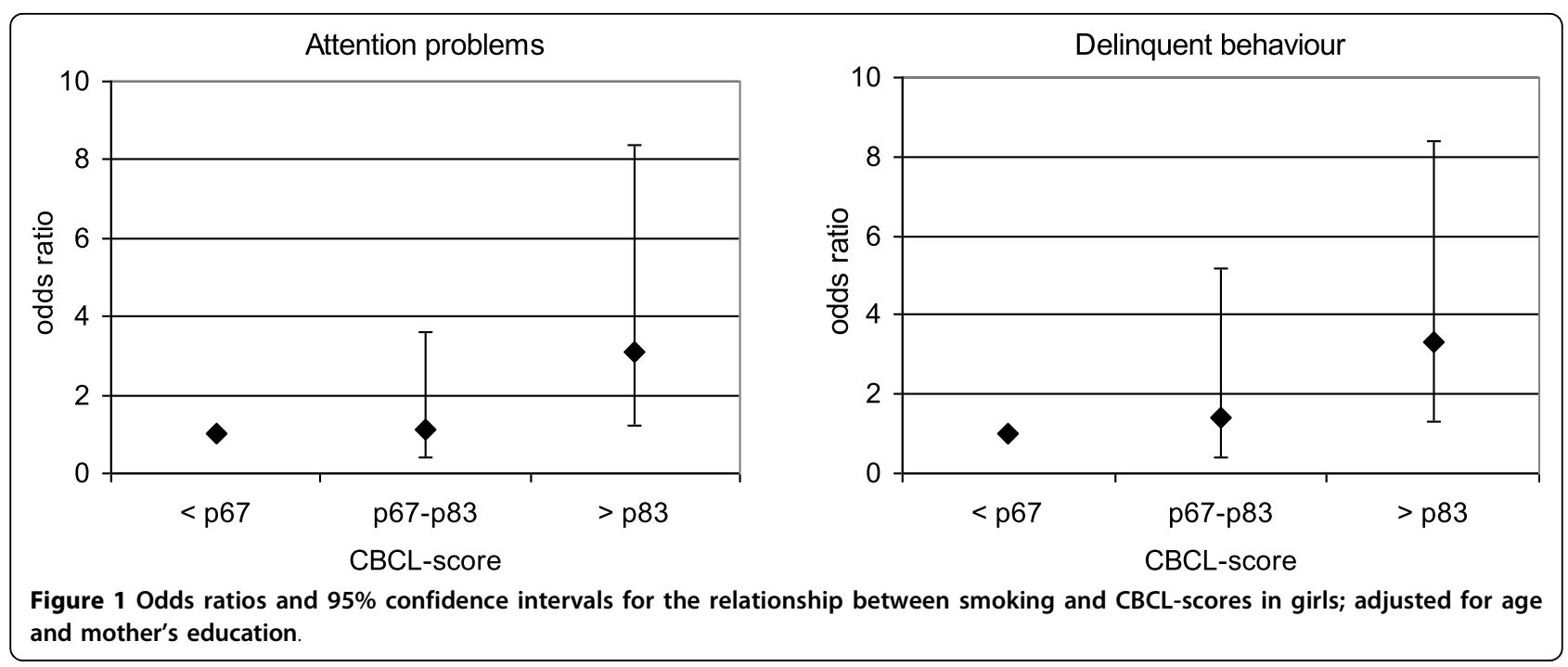

problems search wittingly or unwittingly for relieve of symptoms, for which smoking is rather easy available. Follow-up studies are needed to get insight in the mechanisms of smoking initiation and continuation in children with emotional or behavioural problems.

Interestingly, associations between smoking and psychosocial problems were observed in girls only, and not in boys. We do not have a clear explanation for this finding, though it confirms results of other studies that similarly found a stronger association between smoking and psychopathology in girls $[4,12,17]$. Selective refusal to report smoking is unlikely, neither among boys with problem behaviour nor among girls without problem behaviour. Similarly, it is unlikely that parents of smoking girls were more likely to identify problem behaviour by CBCL compared to parents of smoking boys. An explanation of our gender dependent findings may be a larger proportion of girls having started puberty at age 8-12 years compared to boys, with a concurrent rise in behavioural and emotional problems among girls.

The main limitation of our study is the small number of children that smoked. The potential for further interpretation is limited due to lack of statistical power and allow statistical analysis taking into account multiple confounders. In this study, we therefore limited multiple logistic regression analyses to age, gender, and variables for which frequencies differed statistically significant between smokers and non-smokers, i.e. mother's education. Due to the low number of regular smokers, we limited statistical analyses to starters. In this study, we relied on a self-report of smoking. One might wonder if this introduces information bias. Though, previous studies have shown good validity for self-report of smoking [29].

For the current analyses, data were available for $78.0 \%$ of the $79.0 \%$ parent-child couples that agreed to participate in the study (i.e. $62 \%$ of the invited population). We do not think this has importantly influenced our results, since participants and non-participants did not differ in background characteristics.

Causal inference from our study results is limited due to the cross-sectional design which does not allow inference on the order of smoking and behavioural problems. From previous follow-up studies we assume that psychopathology precedes smoking in at least a substantial proportion of adolescents $[4,5,9,12]$. Large-scale, long-term follow-up studies from early childhood to adulthood will unravel etiological relationships between behavioural problems in smoking trajectories of experimentation, continuation, and regular use.

In summary, in this study we found that the uptake of smoking in pre-adolescent and early adolescent girls occurred more frequently in those with a higher level of psychosocial problems as reported by their parents. Since the relationship was present only for the uppermost $16.7 \%$ of CBCL scores, which agrees with subclinical and clinical scores. Our results suggest that girls in their early teens with problem behaviour may benefit from targeted smoking prevention.

\section{Conclusion}

Among pre-adolescent girls, aged 8-13 years, problem behaviour is associated with the uptake of smoking. Among boys of this age, smoking was not associated with problem behaviour.

\section{Acknowledgements}

The authors acknowledge the Netherlands Social \& Cultural Planning Office (SCP) for financial support of the study, TNO for facilities to perform data collection and statistical analyses. 


\section{Author details}

${ }^{1}$ Municipal Health Service Fryslân, Leeuwarden, the Netherlands, ${ }^{2}$ Department of Health Sciences, University Medical Center Groningen (UMCG), University of Groningen, Groningen, the Netherlands. ${ }^{3}$ TNO (Netherlands Organisation of Applied Scientific Research), Quality of Life, Leiden, the Netherlands.

\section{Authors' contributions}

GM conceived the research question for this paper, performed statistical analyses, and wrote the paper. MR participated in the study design and protocol, supervised data collection and data management. SAR was the principle investigator of this study and conceived the study and financial support. The final manuscript was discussed with all authors, and edited as recommended by co-authors. All authors read and approved the final manuscript.

\section{Competing interests}

The authors declare that they have no competing interests.

Received: 25 November 2009 Accepted: 18 October 2010 Published: 18 October 2010

\section{References}

1. Murray CJ, Lopez AD: On the comparable quantification of health risks: Lessons from the global burden of disease. Epidemiology 1999, 10:594-605.

2. Chassin L, Presson CC, Rose JS, Sherman SJ: The natural history of cigarette smoking from adolescence to adulthood: Demographic predictors of continuity and change. Health Psychol 1996, 15:478-484.

3. Breslau N, Fenn N, Peterson EL: Early smoking initiation and nicotine dependence in a cohort of young adults. Drug Alcohol Depend 1993, 33:129-137.

4. Costello EJ, Erkanli A, Federman E, Angold A: Development of psychiatric comorbidity with substance abuse in adolescents: Effects of timing and sex. J Clin Child Psychol 1999, 28:298-311.

5. Dierker LC, Avenevoli S, Goldberg A, Glantz M: Defining subgroups of adolescents at risk for experimental and regular smoking. Prev Sci 2004, 5:169-183

6. Dierker LC, Avenevoli S, Merikangas KR, Flaherty BP, Stolar M: Association between psychiatric disorders and the progression of tobacco use behaviors. J Am Acad Child Adolesc Psychiatr 2001, 40:1159-1167.

7. Clark DB, Cornelis J: Childhood psychopathology and adolescent cigarette smoking: A prospective survival analysis in children at high risk for substance use disorders. Addict Behav 2004, 29:837-841.

8. Ferdinand RF, Blüm M, Verhulst FC: Psychopathology in adolescence predicts substance use in young adulthood. Addiction 2001, 96:861-870.

9. Crone MR, Reijneveld SA: The association of behavioural and emotional problems with tobacco use in adolescence. Addict Behav 2007, 32:1992-1998

10. De Vries H, Mudde A, Leijs I, Charlton A, Vartiainen E, Buijs G, Clemente MP, Storm H, González Navarro A, Nebot M, Prins T, Kremers S: The European Smoking Prevention Framework Approach (EFSA): an example of integral prevention. Health Educ Res 2003, 18:611-626.

11. Rodham K, Hawton K, Evans E, Weatherall R: Ethnic and gender differences in drinking, smoking and drug taking among adolescents in England: a self-report school-based survey of 15 and 16 year olds. $J$ Adolesc 2005, 28:63-73.

12. Rohde P, Lewinsohn PM, Brown RA, Gau JM, Kahler W: Psychiatric disorders, familial factors and cigarette smoking: I. Associations with smoking initiation. Nicotine Tobacco Research 2003, 5:85-98.

13. Reijneveld SA, Verloove-Vanhorick SP, Hirasing RA: Preventive child healthcare (Jeugdgezondheidszorg). In Volksgezondheid en gezondheidszorg. Edited by: Mackenbach JP, van der Maas PJ. Elsevier gezondheidszorg, Maarsen; 2004:150-160.

14. Achenbach TM: Manual for the CBCL/4-18. Burlington (VT): University of Vermont 2001

15. Verhulst FC, van der Ende J, Koot HM: Manual for the CBCL/4-18 (in Dutch). Rotterdam: Erasmus University 1996.

16. Riggs PD, Mikulich SK, Whitmore EA, Crowley TJ: Relationship of ADHD, depression, and non-tobacco substance use disorders to nicotine dependence in substance-dependant delinquents. Drug Alcohol Depend 1999, 54:195-205.

17. Galéria C, Fombonne E, Chastang JF, Bouvard M: Childhood hyperactivityinattention symptoms and smoking in adolescence. Drug Alcohol Depend 2005, 76:101-108.

18. Juon HS, Ensminger ME, Dobson Sydnor K: A longitudinal study of developmental trajectories to young adult cigarette smoking. Drug Alcohol Depend 2002, 66:303-314.

19. Storr CL, Reboussin BA, Anthony JC: Early childhood misbehavior and the estimated risk of becoming tobacco-dependant. Am J Epidemiol 2004, 160:126-130.

20. Weiser M, Reichenberg A, Grotto I, Yasvitzky R, Rabinowitz J, Lubin G, Nahon D, Knobler HY, Davidson M: Higher rates of cigarette smoking in male adolescents before the onset of schizophrenia: a historicalprospective cohort study. Am J Psychiatry 2004, 161:1219-1223.

21. Biglan A, Duncan TE, Ary DV, Smolkowski K: Peer and parental influences on adolescent tobacco use. J Behav Med 1995, 18:315-330.

22. Levin ED, Conners CK, Silva D, Canu W, March J: Effects of chronic nicotine and methylphenidate in adults with ADHD. Exp Clin Psychopharmacol 2001, 9:83-90.

23. Newhouse PA, Potter A, Singh A: Effects of nicotinic stimulation on cognitive performance. Curr Opin Pharmacol 2004, 4:36-46.

24. Poltavski DV, Petros T: Effects of transdermal nicotine on attention in adult non-smokers with and without attentional deficits. Physiol Behav 2006, 87:614-624.

25. Kumari V, Postma P: Nicotine use in schizophrenia: The self medication hypotheses. Neurosci Behav Rev 2005, 29:1021-1034.

26. Smith RC, Warner-Cohen J, Matute M, Butler E, Kelly E, Vaidhyanathaswamy S, Khan A: Effects of nicotine nasal spray on cognitive function in schizophrenia. Neuropsychopahrmacology 2006, 31:637-643.

27. Milberger S, Biederman J, Faraone SV, Chen L, Jones J: ADHD is associated with early initiation of cigarette smoking in children and adolescents. J Am Acad Child Adolesc Psychiatry 1997, 36:37-44.

28. White HR, Pandina RJ, Chen P-H: Developmental trajectories of cigarette use from early adolescence into young adulthood. Drug Alcohol Depend 2002, 65:167-178.

29. Rebagliato M: Validation of self reported smoking. J Epidemiol Community Health 2001, 56:163-164.

\section{Pre-publication history}

The pre-publication history for this paper can be accessed here: http://www.biomedcentral.com/1471-2458/10/615/prepub

\section{doi:10.1186/1471-2458-10-615}

Cite this article as: de Meer et al:: Gender differences in the association between pre-adolescent smoking initiation and emotional or behavioural problems. BMC Public Health 2010 10:615.

\section{Submit your next manuscript to BioMed Central and take full advantage of:}

- Convenient online submission

- Thorough peer review

- No space constraints or color figure charges

- Immediate publication on acceptance

- Inclusion in PubMed, CAS, Scopus and Google Scholar

- Research which is freely available for redistribution

Submit your manuscript at www.biomedcentral.com/submit
C Biomed Central 